\title{
CFD modelling of weld pool formation and solidification in a laser microwelding process
}

\begin{abstract}
The application of developed thermal models has demonstrated that parameters, such as power, scanning velocity and spot diameter of laser beams have considerable effects on the formation of weld pools. The properties of the weld metal are heavily dependent on the solidification microstructure, and an accurate prediction of the weld pool solidification requires consideration in both the thermodynamics and kinetics of solidification. The computations we presented for a transient three-dimensional model show the aspects of weld pool formation and solidification in a quantitative manner. Our focus was the examination of heat transfer and fluid flow analysis in laser micro-welding of thin stainless-steel sheet (SUS304) using the computational fluid dynamics (CFD) approach. In this research work, a useful linkage between the laser micro-welding parameters and the geometry of the microweld can be derived from the results, and informative guidance was achieved as to how the width, depth and length of the weld pool differ during laser micro-welding as a function of spot diameter, scanning velocity and laser power. The simulation results have been compared with two sets of experimental data to predict the weld bead geometry and solidification pattern made on thin stainless steel sheet using a continuous wave $(\mathrm{CW})$ fibre laser. The reasonable agreement between the simulated and experimental results, demonstrates the reliability of the computed model, and the results can be used to determine the laser microwelding conditions necessary to achieve an appropriate target microstructure. However, the results allow estimation of acceptable ranges of welding variables, to attain the required micro-weld geometry.
\end{abstract}

Keyword: Laser Micro-Welding; Computational Fluid Dynamics (CFD); Heat transfer and Fluid Flow Analysis; Weld Pool Formation; Solidification; Thin Stainless Steel Sheet SUS304 